\title{
Asymptomatic primary tumour in incurable metastatic colorectal cancer: is there a role for surgical resection prior to systematic therapy or not?
}

\author{
Narimantas E. Samalavicius ${ }^{1}$, Audrius Dulskas², Edita Baltruskeviciene ${ }^{3}$, Giedre Smailyte ${ }^{4}$, Marija Skuciene ${ }^{3}$, \\ Rasa Mikelenaite ${ }^{5}$, Rasa Venslovaite ${ }^{3}$, Eduardas Aleknavicius ${ }^{3}$, Almantas Samalavicius ${ }^{6}$, Raimundas Lunevicius ${ }^{7}$ \\ ${ }^{1}$ Center of Oncosurgery, National Cancer Institute, Clinic of Internal, Family Medicine and Oncology, Faculty of Medicine, Vilnius, Lithuania \\ ${ }^{2}$ Center of Oncosurgery, National Cancer Institute, Vilnius, Lithuania \\ ${ }^{3}$ Center of Radiation and Medical Oncology, National Cancer Institute, Vilnius, Lithuania \\ ${ }^{4}$ Centre of Cancer Control and Prevention, National Cancer Institute, Vilnius, Lithuania \\ ${ }^{5}$ Medical Faculty of Vilnius University, Vilnius, Lithuania \\ ${ }^{6}$ Department of Architectural Fundamentals and Theory, Vilnius Gediminas Technical University, Vilnius, Lithuania \\ ${ }^{7}$ General Surgery Department, Aintree University Hospital NHS Foundation Trust, University of Liverpool, Lower Lane, Liverpool, \\ United Kingdom
}

Videosurgery Miniinv 2016; 11 (4): 274-282 DOI: https://doi.org/10.5114/wiitm.2016.64981

\begin{abstract}
Introduction: The role of the resection of asymptomatic primary colorectal cancer in patients with incurable disease is questionable.

Aim: To evaluate the impact of the resection of asymptomatic primary tumour on overall survival in patients with unresectable distant metastases.

Material and methods: Patients treated in the National Cancer Institute, Lithuania, in the period 2008-2012, were selected retrospectively. The main inclusion criteria were: metastatic colorectal cancer $(m C R C)$, endoscopically and histologically confirmed adenocarcinoma, without any symptoms for urgent operation, and at least one cycle of palliative chemotherapy administered. Information on patients' age, gender, tumour histology, localization of the tumour, regional lymph node involvement, number of metastatic sites, surgery and systemic treatment was collected prospectively. Eligible patients for the study were divided into two groups according to the initial treatment - surgery (patients who underwent primary tumour resection) and chemotherapy (patients who received chemotherapy without surgery). The impact of initial treatment strategy, tumour size and site, regional lymph nodes, grade of differentiation of adenocarcinoma and application of biotherapy on overall cumulative survival was estimated using the Kaplan-Meier method. To compare survival between groups the log-rank test was used. Cox regression analysis was employed to assess the effects of variables on patient survival.

Results: The study group consisted of 183 patients: 103 men and 80 women. The median age was 66 years (range: 37-91). There were no notable imbalances with regard to age, gender, number of metastatic sites, metastases (such as pulmonary, peritoneal, liver, metastases into non-regional lymph nodes and other metastases), the number of received cycles of chemotherapy, first line chemotherapy type or biological therapy. Only 27 (14.8\%) patients received biological therapy and the majority of them $(n=25,92.6 \%)$ were treated with bevacizumab. For surgically treated patients 1-year survival was $71.2 \%$ (95\% Cl: 62.1-78.5) and 5-year survival was $4.0 \%$ (95\% Cl: 1.0-10.5). In the chemotherapy group, survival rates were lower -43.9\% (95\% Cl: 31.4-55.7) and 1.7\% (95\% Cl: 0.1-8.1), respectively.
\end{abstract}

Address for correspondence

Audrius Dulskas, National Cancer Institute, 1 Santariskiu St, LT-08406 Vilnius, Lithuania, phone: +37 067520094 ,

e-mail: audrius.dulskas@gmail.com 
Better survival rates were in the palliative surgery group. Multivariate analysis using the Cox proportional hazards model revealed that the initial palliative surgery and the application of biological therapy were statistically significant independent prognostic factors for survival.

Conclusions: Our findings suggest that palliative resectional surgery for the primary tumour in patients with incurable $m C R C$ improves survival. Of course, one can argue that patients in the surgery group were "less problematic". Prospective randomized trials are needed to delineate precisely the role of palliative surgery of the primary tumour in these patients.

Key words: primary tumour, metastatic colorectal cancer, resection, chemotherapy, survival.

\section{Introduction}

Globally, colon and rectum cancer (CRC) ranked third for cancer incidence and fourth for cancer death in 2013. For developed countries it ranked second for incidence and mortality, and in developing countries it ranked fourth for both incidence and mortality [1]. There is a tendency of mortality decrease [2].

Approximately $25 \%$ of patients present with metastases (MTS) at initial diagnosis [3]. According to the guidelines provided by the National Comprehensive Cancer Network (NCCN), in patients with unresectable metastases and an intact primary tumour that is not obstructing the bowel, systemic chemotherapy is the favoured initial step of treatment [4]. Resection of a primary tumour is required only to palliate symptoms related to obstruction, perforation, or bleeding [5].

However, the effect of primary tumour resection on survival remains unclear. In 2012, the Cochrane collaboration analysed 7 non-randomized clinical studies. These trials included 1,086 patients (722 patients were treated with primary tumour resection and 364 managed first with chemotherapy and/or radiotherapy) [6]. There was no difference in overall survival between those two groups. In addition, it was reported that resection does not prevent intraabdominal complications. Nevertheless, some studies have demonstrated a benefit of the surgical resection of the primary tumour $[7,8]$. Therefore, prospective randomised trials are warranted to investigate the need of surgery in these patients. Currently, the results are awaited from randomized, multi-institutional phase III studies, such European CAIRO4 [9] and SYNCHRONOUS [10], or the Korean NCT01978249 trial [11]. Furthermore, the ORCHESTRA trial [12] a multicentre randomized controlled trial (RCT) investigating the combination of chemotherapy and maximal tumour debulking compared with chemotherapy alone in patients with multiorgan CRC metastases - could contribute interesting data.

\section{Aim}

The aim of our retrospective study was to review our institution's experience while treating colorectal cancer patients with incurable distant disease, and to evaluate the benefit of resection of the asymptomatic primary tumour prior to systemic therapy.

\section{Material and methods}

All patients with stage IV colorectal cancer treated at the Lithuanian National Cancer Institute from January $1^{\text {st }}, 2008$ to December $31^{\text {st }}, 2012$ were evaluated retrospectively. Patients with clinically (metastases) and histologically (primary tumour) verified stage IV colorectal cancer (C18, C19 and C20 in International Classification of Diseases (ICD)-10) with asymptomatic primary tumour, and at least one cycle of palliative chemotherapy (FOLFOX, FOLFIRI or fluoropyrimidine, including the De Gramont and Mayo regimen or capecitabine) were included in this study.

Information on patients' age, gender, tumour histology, localization of the tumour, regional lymph node involvement, number of metastatic sites, surgery and systemic treatment was collated. Eligible patients for the study were divided into two groups according to the initial treatment - surgery (patients who underwent primary tumour resection) and chemotherapy (patients who received chemotherapy without surgery).

\section{Statistical analysis}

The impact of initial treatment strategy, tumour size, regional lymph nodes, grade of differentiation of adenocarcinoma and application of biotherapy on overall cumulative survival was estimated using the Kaplan-Meier method. To compare survival between 
groups the log-rank test was used. Cox regression analysis was employed to assess the effects of variables on patient survival. All statistical analyses were performed using Stata Statistical Software version 11.0 (StataCorp. 2009. Stata Statistical Software: Release 11.0. College Station, TX, USA).

\section{Results}

\section{Study group characteristics}

A total of 300 patients diagnosed with stage IV colorectal cancer were selected. One hundred and seventeen patients, who had a symptomatic tumour (bowel obstruction, perforation or bleeding), did not receive chemotherapy and/or who were scheduled for radical resection of metastases were not included in the study group. The study group consisted of 183 patients: 103 men and 80 women. The median age was 66 years (range: 37-91). There were $120 \mathrm{pa}$ tients included in the surgery arm and 63 patients in the chemotherapy arm.

Table I provides information on baseline characteristics of 183 patients broken down by the initial management of the patients. There were no notable imbalances with regard to age, gender, number of metastatic sites, metastases (such as pulmonary, peritoneal, liver, metastases into non-regional lymph nodes and other metastases), and the number of received cycles of chemotherapy, first line chemotherapy type or biological therapy. A significant difference was observed in tumour site (more colon cancers occurred in the surgery group), and histological type (more adenocarcinomas with a mucinous component were also observed in the surgery group). One hundred and thirty-eight (75.4\%) patients received 1-2 cycles of chemotherapy, while the rest 45 (24.6\%) received 3 or more cycles of chemotherapy. The most popular type of chemotherapy was fluoropyrimidines. As the first line therapy fluoropyrimidines were administered to 94 (51.4\%) patients, while FOLFOX was administered to 68 (37.2\%) patients and FOLFIRI to 21 (11.5\%) patients. The median number of administered cycles was 6 (range: 1-12). Only 27 (14.8\%) patients received biological therapy, and the majority of them $(n=25$, 92.6\%) were treated with bevacizumab.

\section{Treatment outcomes}

For those patients who underwent resection of the primary tumour, 1-year survival was $71.2 \%$ (95\%
Cl: 62.1-78.5), 2-year survival 24.5\% (95\% Cl: 17.132.6), 3-year survival $10.3 \%(95 \% \mathrm{Cl}: 5.5-16.7)$ and 5-year survival $4.0 \%(95 \% \mathrm{Cl}: 1.0-10.5)$. In the chemotherapy group survival rates were lower $-43.9 \%$ (95\% Cl: 31.4-55.7), 15.6\% (95\% Cl: 7.8-25.6), 1.7\% (95\% Cl: $0.1-8.1)$ and $1.7 \%(95 \% \mathrm{Cl}: 0.1-8.1)$, respectively. Kaplan-Meier survival analysis showed no statistically significant differences between patients with regard to age, gender, number of metastatic sites, metastases (such as pulmonary, peritoneal, liver, metastases into non-regional lymph nodes and other metastases), and first line chemotherapy type. In contrast, initial surgical treatment was associated with significantly better survival (Figure 1). Analysis of survival in the group of surgically treated patients separately showed the same tendency according to regional lymph node involvement, but survival differences between groups were not significant, probably because of the smaller group size. Grade of differentiation, tumour location in the rectum and biologic therapy were other significant factors for better survival of colorectal cancer patients (Figures 2-5). Multivariate analysis using Cox's proportional hazards model revealed that the initial palliative surgery ( $p=0.02,95 \% \mathrm{Cl}: 1.06-2.41)$ site of the tumour in the rectum ( $p=0.015,95 \% \mathrm{Cl}: 0.48-0.92)$ and the application of biological therapy ( $p=0.01,95 \% \mathrm{Cl}$ : 1.14-3.02) were statistically significant independent prognostic factors for better survival (Table II).

\section{Discussion}

Traditionally, treatment plans for metastatic colorectal cancer depend on symptoms of obstruction, perforation, and haemorrhage from the primary tumour, resectability of metastases and the patient's performance status. In this study, we included only asymptomatic patients and demonstrated that palliative resection of primary tumour is an independent prognostic factor for statistically significant better overall survival.

Many surgeons support resection of the primary tumour to prevent local tumour complications. Several studies that used registry data have demonstrated a survival benefit of resection of the primary tumour in stage IV CRC [7, 8, 13-22]. However, most studies failed to adjust for performance status and other important clinicopathological variables. Recent studies from Canada showed improved overall survival in asymptomatic patients undergoing palliative resection [8, 14]. After adjustment for age, performance 
Table I. Baseline characteristics of patients according to initial treatment

\begin{tabular}{|c|c|c|c|c|c|c|}
\hline \multirow[t]{2}{*}{ Parameter } & \multirow[t]{2}{*}{$N$} & \multicolumn{2}{|c|}{ Chemotherapy } & \multicolumn{2}{|c|}{ Surgery } & \multirow[t]{2}{*}{$P$-value } \\
\hline & & $n$ & $\%$ & $n$ & $\%$ & \\
\hline Total & 183 & 63 & 34.4 & 120 & 65.6 & - \\
\hline \multicolumn{7}{|l|}{ Age: } \\
\hline$<70$ & 119 & 43 & 36.1 & 76 & 63.9 & \multirow[t]{2}{*}{0.507} \\
\hline$\geq 70$ & 64 & 20 & 31.3 & 44 & 68.8 & \\
\hline \multicolumn{7}{|l|}{ Gender: } \\
\hline Female & 79 & 26 & 32.9 & 53 & 67.1 & \multirow[t]{2}{*}{0.707} \\
\hline Male & 104 & 37 & 35.6 & 67 & 64.4 & \\
\hline \multicolumn{7}{|l|}{ Tumour site: } \\
\hline Colon (C18-C19) & 96 & 25 & 26.0 & 71 & 74.0 & \multirow[t]{2}{*}{0.012} \\
\hline Rectum (C20) & 87 & 38 & 43.7 & 49 & 56.3 & \\
\hline \multicolumn{7}{|l|}{ Tumour differentiation grade: } \\
\hline G1-G2 & 156 & 55 & 35.3 & 101 & 64.7 & \multirow[t]{3}{*}{0.831} \\
\hline G3 & 21 & 6 & 28.6 & 15 & 71.4 & \\
\hline Not available & 6 & 2 & 33.3 & 4 & 66.6 & \\
\hline \multicolumn{7}{|l|}{ Histological tumour type: } \\
\hline Adenocarcinoma & 166 & 61 & 36.7 & 105 & 63.3 & \multirow[t]{2}{*}{0.039} \\
\hline Adenocarcinoma with mucinous differentiation & 17 & 2 & 11.8 & 15 & 88.2 & \\
\hline \multicolumn{7}{|l|}{ Number of metastatic sites: } \\
\hline 1 & 117 & 41 & 35.0 & 76 & 65.0 & \multirow[t]{2}{*}{0.815} \\
\hline$\geq 2$ & 66 & 22 & 33.3 & 44 & 66.67 & \\
\hline \multicolumn{7}{|l|}{ Liver metastases: } \\
\hline Yes & 147 & 46 & 31.3 & 102 & 69.4 & \multirow[t]{2}{*}{0.071} \\
\hline No & 36 & 17 & 47.2 & 19 & 52.8 & \\
\hline \multicolumn{7}{|l|}{ Pulmonary metastases: } \\
\hline Yes & 35 & 15 & 42.9 & 20 & 57.1 & \multirow[t]{2}{*}{0.243} \\
\hline No & 148 & 48 & 32.4 & 101 & 67.6 & \\
\hline \multicolumn{7}{|l|}{ Peritoneal metastases: } \\
\hline Yes & 18 & 7 & 38.9 & 11 & 61.1 & \multirow[t]{2}{*}{0.675} \\
\hline No & 165 & 56 & 33.9 & 109 & 66.1 & \\
\hline \multicolumn{7}{|l|}{ Metastases into non-regional lymph nodes: } \\
\hline Yes & 29 & 10 & 34.5 & 19 & 65.5 & \multirow[t]{2}{*}{1.000} \\
\hline No & 154 & 53 & 34.4 & 101 & 65.6 & \\
\hline
\end{tabular}


Table I. Cont.

\begin{tabular}{|c|c|c|c|c|c|c|}
\hline \multirow[t]{2}{*}{ Parameter } & \multirow[t]{2}{*}{$N$} & \multicolumn{2}{|c|}{ Chemotherapy } & \multicolumn{2}{|c|}{ Surgery } & \multirow[t]{2}{*}{$P$-value } \\
\hline & & $n$ & $\%$ & $n$ & $\%$ & \\
\hline \multicolumn{7}{|c|}{ Number of received cycles of chemotherapy: } \\
\hline $1-2$ & 138 & 51 & 37.0 & 88 & 63.0 & \multirow[t]{2}{*}{0.207} \\
\hline$\geq 3$ & 45 & 12 & 26.7 & 33 & 73.3 & \\
\hline \multicolumn{7}{|c|}{ First line chemotherapy type: } \\
\hline FOLFOX & 68 & 17 & 25.0 & 51 & 75.0 & \multirow[t]{3}{*}{0.114} \\
\hline FOLFIRI & 21 & 9 & 42.9 & 12 & 57.1 & \\
\hline Fluoropyrimidines & 94 & 37 & 39.4 & 57 & 60.1 & \\
\hline \multicolumn{7}{|c|}{ First line biological therapy: } \\
\hline Yes & 27 & 7 & 25.9 & 20 & 74.1 & \multirow[t]{2}{*}{0.314} \\
\hline No & 156 & 56 & 35.9 & 101 & 64.8 & \\
\hline
\end{tabular}

$N$ - patient number.

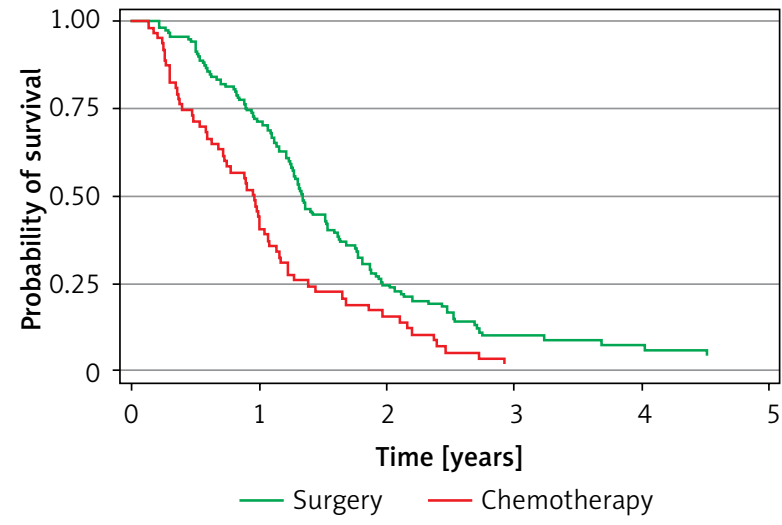

Figure 1. The Kaplan-Meier analysis of overall survival by the initial type of treatment $(p=0.0007)$

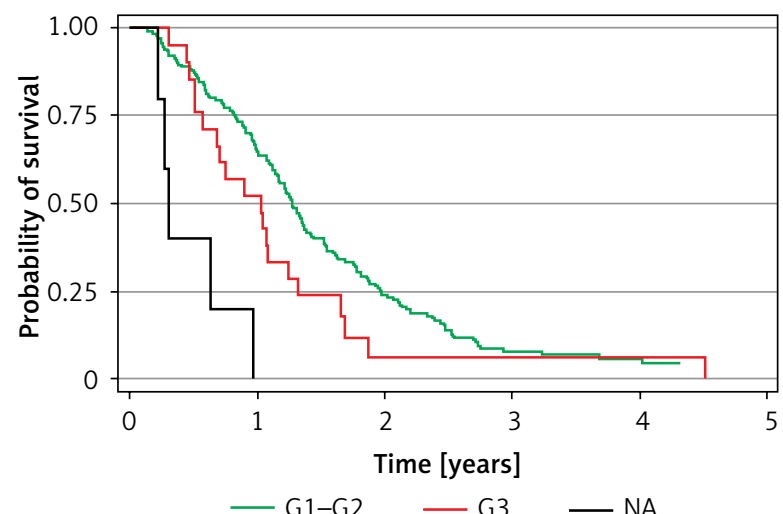

Figure 3. The Kaplan-Meier analysis of overall survival by grade of differentiation $(p<0.0001)$

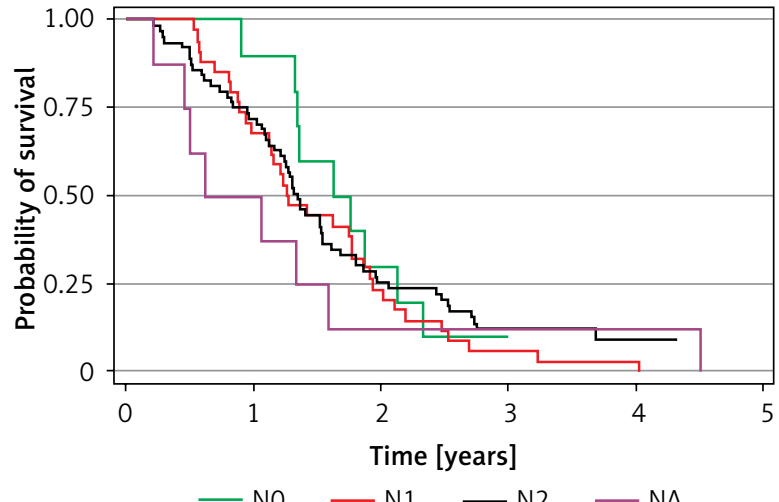

Figure 2. The Kaplan-Meier analysis of overall survival by regional lymph node involvement in the surgically treated patients $(p=0.48)$

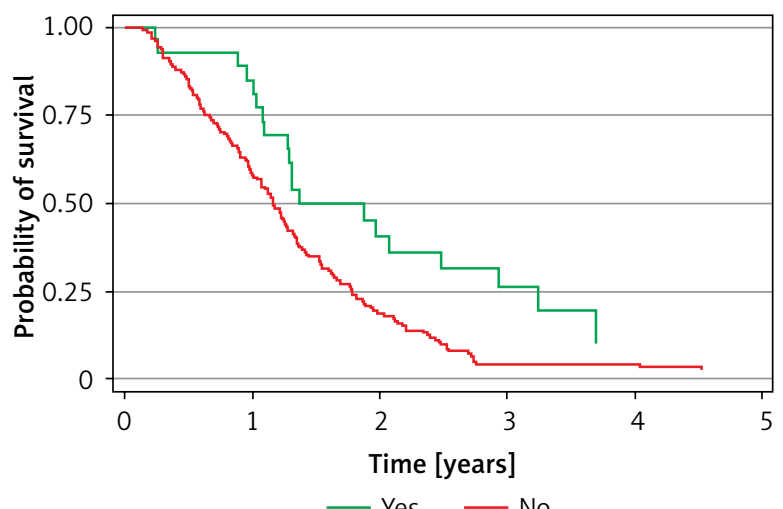

Figure 4. The Kaplan-Meier analysis of overall survival by biological therapy (applied/not applied) $(p=0.007)$ 
status, comorbid illnesses, and chemotherapy, resection of the primary tumour in patients with stage IV CRC and asymptomatic or minimally symptomatic primary tumour improves survival. The unadjusted survival difference between the groups with surgery versus no surgery was 11.3 months. Ahn et al. in their study showed that even in elderly stage IVb patients primary cancer resection improves survival [15]. In a small study from Japan the authors found that even in stage IV CRC, patients benefit from D3 lymph node dissection [19]. In a single centre retrospective study from Korea the authors assessed colorectal patients with unresectable liver MTS (no other sites of MTS). The first group, comprising 195 patients who underwent primary tumour resection, was compared with the second group of 66 patients without surgery. Overall survival (OS) was 21 and 10 months respectively $(p<0.001)$ [20]. In another large multicentre retrospective study from Japan with almost 2000 patients included, a significant

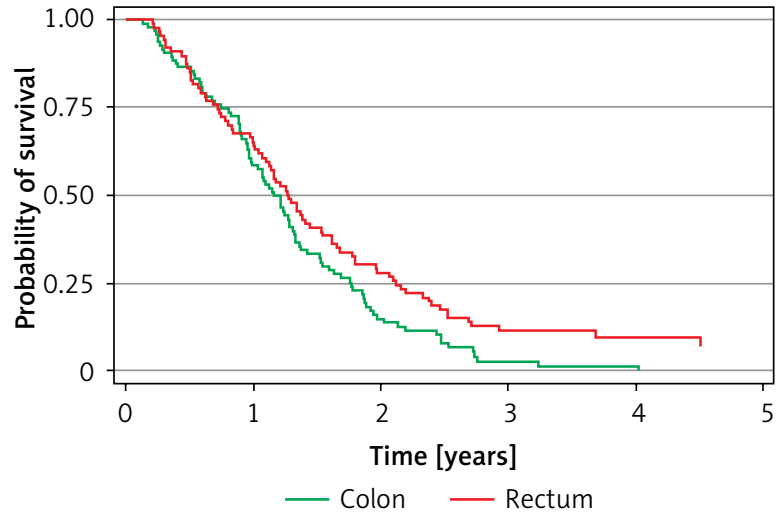

Figure 5. The Kaplan-Meier analysis of overall survival by tumour site (applied/not applied) $(p=0.0364)$

cancer-specific survival improvement was found in all the primary tumour resection group. In subgroup analysis no benefit in older patients (more than 65 years), patients with nodal involvement and in

Table II. Cox multivariate analysis between four variables and survival of the patients with incurable metastatic colorectal cancer

\begin{tabular}{|c|c|c|c|}
\hline Parameter & Hazard ratio & $95 \%$ confidence interval & $P$-value \\
\hline \multicolumn{4}{|l|}{ Initial treatment*: } \\
\hline Surgery & Ref. & - & - \\
\hline Chemotherapy & 1.76 & $1.16-2.68$ & 0.008 \\
\hline \multicolumn{4}{|l|}{ Tumour site*: } \\
\hline Colon & Ref. & - & - \\
\hline Rectum & 0.67 & $0.48-0.92$ & 0.015 \\
\hline \multicolumn{4}{|c|}{ Regional lymph nodes: } \\
\hline NO & Ref. & - & - \\
\hline $\mathrm{N} 1$ & 1.79 & $0.90-3.53$ & 0.09 \\
\hline N2 & 2.02 & $1.03-3.93$ & 0.04 \\
\hline NA & 1.97 & $0.98-3.96$ & 0.06 \\
\hline \multicolumn{4}{|c|}{ Grade of differentiation: } \\
\hline $\mathrm{G} 1-\mathrm{G} 2$ & Ref. & - & - \\
\hline G3 & 1.45 & $0.89-2.34$ & 0.13 \\
\hline NA & 4.04 & $1.54-10.59$ & 0.004 \\
\hline \multicolumn{4}{|l|}{ Biotherapy*: } \\
\hline Applied & Ref. & - & - \\
\hline Not applied & 1.84 & $1.13-2.98$ & 0.01 \\
\hline
\end{tabular}

*Statistically significant independent prognostic factors. 
right-sided tumours was found [21]. Tarantino et al. recently contributed to this discussion by reporting the largest observational study conducted to date [22]. They analysed the population-based data from 37,793 patients with mCRC. The results of the univariable Cox regression analysis after stratified propensity score matching showed a hazard ratio for overall survival of $0.40(95 \% \mathrm{Cl}$ : $0.39-0.42$; $p<0.001)$ and for cancer-specific survival of 0.39 (95\% Cl: $0.38-0.40 ; p<0.001)$, in favour of patients who underwent resection.

However, there are opinions that, in patients with an asymptomatic advanced $\mathrm{mCRC}$, the first treatment choice should not be removing the primary tumour, because these patients mainly die of systemic disease, rather than complications associated with the primary cancer [6, 23-28]. Our already mentioned systemic review and meta-analysis performed by Cirocchi et al. did not show any difference in survival [6]. Several factors may explain these disparate results. Foremost, different selection criteria were used across studies. After a careful systemic review based on very strict inclusion criteria, only seven articles were included in the final analysis. In addition, the authors were able to report on outcomes in only four of the seven studies that had survival data available, of which half the studies favoured primary tumour resection and the other half did not find any significant differences in OS. In a small group of 48 patients from Italy, the authors found no difference in OS, but higher risk of all-cause death in the surgery group. It was increased in patients with more than $75 \%$ of liver parenchyma affected [25]. Matsumoto et al. found that the endoscopic traversability during the initial diagnosis is the main factor showing the need of upfront resection [27]. Twenty-five percent of patients in the chemotherapy group required surgery within 1 year. Other risk factors for the need of surgical or endoscopic intervention in the chemotherapy group are tumour location in the rectum and tumour size larger than $5 \mathrm{~cm}$ [28].

There are some other essential questions regarding cancer biology possible change after resection of a primary tumour. For instance, how does tumour biology change without the primary tumour? Do they become more aggressive or vice versa? How does the decrease in overall cancer burden increase overall survival? Some evidence supports that resection of the primary tumour may improve disease control [13]. There is evidence for genetic variation between the primary tumours and metastases [29]. Removal of the primary tumour might result in a decreased burden of chemotactic cytokines and tumour-promoting factors that are produced by CRCs and regulate tumour cell growth and metastasis [30-32]. Alteration in the immune response after surgery, with a predominant Thelper 1 response rather than one with a substantial T helper 2 component, might contribute to a better outcome [33]. A few studies showed that removal of the primary tumour causes reversal of the neutrophil-to-lymphocyte ratio (NLR), which is a good prognostic marker of the systemic inflammatory response. Low or decreased NLR leads to significant improvement of OS [34, 35]. In patients suffering from cancer, muscle mass and aerobic function decline. Removal of the primary tumour showed a significant improvement of aerobic function [36].

Two clinical studies from France suggested that the presence of the primary tumour could be involved in less efficient antitumor activity of antiangiogenic agents [37, 38]. In a small multicentre study from France with 96 patients included after adjustment inverse probability treatment weight results showed longer OS in the primary tumour resection and bevacizumab group [37]. In a larger study with 400 patients Ghiringhelli et al. showed a good effect of bevacizumab on OS only in patients undergoing primary tumour resection [38]. Although only $27 \mathrm{pa}-$ tients in our study received bevacizumab postoperatively (the small number of these patients is due to the difficulties in our health care system), we found similar positive results.

It was pointed out that mucinous histology of adenocarcinoma often determines up-front surgical treatment. However, poor prognosis of this histology type is only present in rectal cancer [39]. In our study, all primary rectal tumours with mucinous histology were in the up-front palliative resection group. Our findings suggest that patients with rectal tumours compared to those who had a tumour in the colon may have better survival. Also it is well known that colon cancer has a better prognosis than rectum cancer [40]. One might argue that this is the reason we had better overall survival rates in the surgery group (there were more stage IV colon cancers than in the chemotherapy group). But, as we mentioned before, in our study rectal cancer patients had better survival rates.

Radiofrequency ablation for liver metastasis may have a certain role even for incurable metastatic col- 
orectal cancer patients [41], but in our study we included only patients who were treated for their disseminated disease only with systemic therapy, regardless of whether the primary tumour was removed.

This study has a few limitations. The findings should be taken into account with caution because of the retrospective design of the study, single institutional review, low sample size, possible selection bias ("less problematic" patients included in the surgery group), no stratification of patients by performance status and combined analysis of colonic and rectal cancers. Also BRAF mutation, which is an important prognostic marker in $\mathrm{MCRC}$, was not assessed. Therefore, larger prospective randomised studies on colon and rectal cancers separately are warranted, for there is still some debate about the optimal standard approach to elective up-front treatment for patients with advanced metastatic stage IV colon or rectal cancer.

\section{Conclusions}

Our findings suggest that palliative resectional surgery for the primary tumour in patients with incurable metastatic colorectal cancer improves survival. Prospective randomized trials are needed to delineate precisely the role of palliative surgery of the primary tumour in these patients.

\section{Conflict of interest}

The authors declare no conflict of interest.

\section{References}

1. Global Burden of Disease Cancer Collaboration. The Global Burden of Cancer 2013. JAMA Oncol 2015; 1: 505-27.

2. Lunevicius R, Poskus T, Samalavicius NE. National burden of colorectal cancer in Lithuania and the ranking of Lithuania within the 45 European nations. Oncol Lett 2015; 10: 433-8.

3. van Cutsem E, Cervantes A, Nordlinger B, Arnold D; the ESMO Guidelines Working Group. Metastatic colorectal cancer: ESMO clinical practice guidelines. Ann Oncol 2014; 25: 1-9.

4. National Comprehensive Cancer Network. NCCN clinical practice guidelines in oncology (NCCN guidelines): colon/rectal cancer. Ver. 2. 2016 [Internet]. Fort Washington: NCCN; c2016 [cited 2016 Mar 20]. Available from: http://www.nccn.org/professionals/physician_gls/pdf/rectal.pdf

5. Poultsides GA, Servais EL, Saltz LB, et al. Outcome of primary tumor in patients with synchronous stage IV colorectal cancer receiving combination chemotherapy without surgery as initial treatment. J Clin Oncol 2009; 27: 3379-84.

6. Cirocchi R, Trastulli S, Abraha I, et al. Non-resection versus resection for an asymptomatic primary tumour in patients with unresectable stage IV colorectal cancer. Cochrane Database Syst Rev 2012; 8: CD008997.

7. Gresham G, Renouf D, Chan M, et al. Association between palliative resection of the primary tumor and overall survival in a population-based cohort of metastatic colorectal cancer patients. Ann Surg Oncol 2014; 21: 3917-23.

8. Ahmed S, Leis A, Fields A, et al. Survival impact of surgical resection of primary tumor in patients with stage IV colorectal cancer. Cancer 2014; 120: 683-91.

9. 't Lam-Boer J, Mol L, Verhoef C, et al. The CAIRO4 study: the role of surgery of the primary tumour with few or absent symptoms in patients with synchronous unresectable metastases of colorectal cancer - a randomized phase III study of the Dutch Colorectal Cancer Group (DCCG). BMC Cancer 2014; 14: 741.

10. Rahbari NN, Lordick F, Fink C, et al. Resection of the primary tumour versus no resection prior to systemic therapy in patients with colon cancer and synchronous unresectable metastases (UICC stage IV): SYNCHRONOUS, a randomised controlled multicentre trial (ISRCTN30964555). BMC Cancer 2012; 12: 142.

11. Kim CW, Baek JH, Choi GS, et al. The role of primary tumor resection in colorectal cancer patients with asymptomatic, synchronous unresectable metastasis: study protocol for a randomized controlled trial. Trials 2016; 17: 34-41.

12. US National Library of Medicine. ClinicalTrials.gov [online], https://clinicaltrials.gov/ct2/show/NCT01792934 (2014).

13. Faron M, Bourredjem A, Pignon J, et al. Impact on survival of primary tumor resection inpatients with colorectal cancer and unresectable metastasis: pooled analysis of individual patients' data from four randomized trials. J Clin Oncol 2012; 30 (Suppl): abstract 3507.

14. Ahmed S, Fields A, Pahwa P, et al. Surgical resection of primary tumor in asymptomatic or minimally symptomatic patients with stage IV colorectal cancer: a Canadian province experience. Clin Colorectal Cancer 2015; 4: 41-7.

15. Ahn HJ, Oh HS, Ahn Y, et al. Prognostic implications of primary tumor resection in stage IVb colorectal cancer in elderly patients. Ann Coloproctol 2014; 30: 175-81.

16. Gresham G, Renouf DJ, Chan M, et al. Association between palliative resection of the primary tumor and overall survival in a population-based cohort of metastatic colorectal cancer patients. Ann Surg Oncol 2014; 12: 3917-23.

17. Chan TW, Brown C, Ho CC, Gill S. Primary tumor resection in patients presenting with metastatic colorectal cancer: analysis of a provincial population-based cohort. Am I Clin Oncol 2010; 33: 52-5.

18. Ishihara S, Hayama T, Yamada H, et al. Prognostic impact of primary tumor resection and lymph node dissection in stage IV colorectal cancer with unresectable metastasis: a propensity score analysis in a multicenter retrospective study. Ann Surg Oncol 2014; 21: 2949-55.

19. Sugihara K, Watanabe T. Prognostic impact of primary tumor resection and lymph node dissection in stage iv colorectal cancer with unresectable metastasis: a propensity score analysis in a multicenter retrospective study. Ann Surg Oncol 2014; 21: 2949-55.

20. Yoon YS, Kim CW, Lim SB, et al. Palliative surgery in patients with unresectable colorectal liver metastases: a propensity score matching analysis. J Surg Oncol 2014; 109: 239-44. 
21. Ishihara S, Nishikawa T, Tanaka T, et al. Benefit of primary tumor resection in stage IV colorectal cancer with unresectable metastasis: a multicenter retrospective study using a propensity score analysis. Int J Colorectal Dis 2015; 30: 807-12.

22. Tarantino I, Warschkow R, Worni $\mathrm{M}$ et al. Prognostic relevance of palliative primary tumor removal in 37,793 metastatic colorectal cancer patients: a population-based, propensity score-adjusted trend analysis. Ann Surg 2015; 262: 112-20.

23. Eisenberg A, Whelan RL, Neugut Al. Survival and symptomatic benefit from palliative primary tumor resection in patients with metastatic colorectal cancer: a review. Int J Colorectal Dis 2008; 23: 559-68.

24. Miyamoto Y, Watanabe M, Sakamoto Y, et al. Evaluation of the necessity of primary tumor resection for synchronous metastatic colorectal cancer. Surgery Today Official Journal of the Japan Surgical Society 2014 10.1007/s00595-014-0869-5.

25. Boselli C, Renzi C, Gemini A, et al. Surgery in asymptomatic patients with colorectal cancer and unresectable liver metastases: the authors' experience. Onco Targets Ther 2013; 6: 267-72.

26. Ichikawa Y, Goto A, Kobayashi N, et al. Does resection of primary lesions show survival benefit for stage IV colorectal cancer patients with unresectable metastases? Hepatogastroenterology 2013; 60: 1945-49.

27. Matsumoto T, Hasegawa S, Matsumoto S, et al. Overcoming the challenges of primary tumor management in patients with metastatic colorectal cancer unresectable for cure and an asymptomatic primary tumor. Dis Colon Rectum 2014; 57: 679-86.

28. Yun JA, Park Y, Huh JW, et al. Risk factors for the requirement of surgical or endoscopic interventions during chemotherapy in patients with uncomplicated colorectal cancer and unresectable synchronous metastases. J Surg Oncol 2014; 110: 839-44.

29. Goranova TE, Ohue M, Shimoharu Y, Kato K. Dynamics of cancer cell subpopulations in primary and metastatic colorectal tumors. Clin Exp Metastasis 2011; 28: 427-35.

30. Cambien B, Richard-Fiardo P, Karimdjee BF, et al. CCL5 neutralization restricts cancer growth and potentiates the targeting of PDGFRb in colorectal carcinoma. PLoS One 2011; 6: e28842.

31. Chen HJ, Edwards R, Tucci S, et al. Chemokine 25-induced signalling suppresses colon cancer invasion and metastasis. J Clin Invest 2012; 122: 3184-96.

32. Merogi AJ, Ramesh R, Robinson WR, et al. Tumor-host interaction: analysis of cytokines, growth factors, and tumor-infiltrating lymphocytes in ovarian carcinomas. Hum Pathol 1997; 28: 321-31.

33. Ahmed S, Wei G, Lim J, et al. Immune response and its correlation with the disease activity in patients with advanced colorectal cancer ( $\mathrm{aCRC}$ ): results from a prospective observational study. J Clin Oncol 2014; 32 (Suppl 3): Abstract 471.

34. Maeda K, Shibutani M, Otani H, et al. Prognostic value of preoperative inflammation-based prognostic scores in patients with stage IV colorectal cancer who undergo palliative resection of asymptomatic primary tumors. Anticancer Res 2013; 33: $5567-73$

35. Turner N, Tran B, Tran PV, et al. Primary tumor resection in patients with metastatic colorectal cancer is associated with reversal of systemic inflammation and improved survival. Clin Colorectal Cancer 2015; 14: 185-91.
36. Williams JP, Nyasavajjala SM, Phillips BE, et al. Surgical resection of primary tumour improves aerobic performance in colorectal cancer. Eur J Surg Oncol 2014; 40: 220-6.

37. de Mestier L, Neuzillet C, Pozet A, et al. Is primary tumor resection associated with a longer survival in colon cancer and unresectable synchronous metastases? A 4-year multicentre experience. Eur J Surg Oncol 2014; 40: 685-91.

38. Ghiringhelli F, Bichard D, Limat S, et al. Bevacizumab efficacy in metastatic colorectal cancer is dependent on primary tumor resection. Ann Surg Oncol 2014; 21: 1632-40.

39. Hugen N, Verhoeven RH, Radema SA, et al. Prognosis and value of adjuvant chemotherapy in stage III mucinous colorectal carcinoma. Ann Oncol 2013; 24: 2819-24.

40. www.cancer.org/cancer/colonandrectumcancer/detailedguide/colorectal-cancer-survival-rates

41. Vavra P, Nowakova J, Ostruszka P, et al. Colorectal cancer liver metastases: laparoscopic and open radiofrequency-assisted surgery. Videosurgery Miniinv 2015; 10: 205-12.

Received: 29.06.2016, accepted: 18.12.2016. 